MUZIKOLOSKI ZBORNIK - MUSICOLOGICAL ANNUAL XIII, LJUBLJANA 1977

UDK 78(45) Carissimi (437)

\title{
GIACOMO CARISSIMIS KOMPOSITIONEN IN DEN BÖHMISCHEN LÄNDERN
}

Jiří Sehnal (Brno)

Giacomo Carissimi gehört zu jenen Meistern der Vergangenheit, die man in den letzten hundert Jahren, je nach der Einstellung der Musikwissenschaftler, mehr oder weniger geschätzt hat. Heute erkennt man aber eindeutig seine Verdienste um die Entstehung des Oratoriums, ${ }^{1}$ der italienischen Kantate und des bel canto ${ }^{2}$ an. Unlängst hat G. Massenkeil ${ }^{3}$ die Berechtigung des Titel Musikrhetor bestätigt, den die Zeitgenossen Ca. rissimi verliehen hatten. Etwas zweifelhaft bleibt allerdings die Wahl der Kompositionsmittel dieses Autors, die beispielsweise im Vergleich mit Monteverdi ärmlich und stereotyp erscheinen. Trotzdem gehört Carissimi zu den echten Musikerpersönlichkeiten des 17. Jahrhunderts, deren Werke eine Gesamtausgabe verdienen würden.4

Giacomo Carissimi verschafften bereits zu Lebzeiten die Aufführungen seiner Oratorien in der Jesuitenkirche San Apollinare zu Rom, wo er seit 1630 wirkte, internationalen Ruf. Während aber seine Messen, Motetten und weltlichen Kantaten in zahlreichen Abschriften und auch im Druck über ganz Europa Verbreitung fanden, blieben die Oratorien eher den Besuchern Roms bekannt und drangen über die Grenzen der ewigen Stadt kaum hinaus. Man erklärt das meist durch das Verbot Papst Klemens des X. (1670-1676), die Musikalien des Collegium Germanicum, des Eigentumers der Kirche San Apollinare und Erben von Carissimis kompositorischen Vermächtnis, abzuschreiben und zu verleihen. Angeblich existierte

1 Die besten Wertungen der Oratorien Carissimis brachten besonders: Brenet M., Les "Oratorios" de Carissimi, PMI 4, 1897, 460 ff.; Pratella B., Giacomo Carissimi ed $i$ suoi Oratori, RMI 27, 1920, 1 ff.; Bukofzer M. F., Music in the Baroque Era, New Yoris 1947, 120 ff.; Ghisi F., Giacomo Carissimi, in: La Musica, Enciclopedia storica Vol. 1, Torino 1966, $797 \mathrm{ff}$.

2 Landshoff L., Nachwort zu Alte Meister des Bel canto, Bd. 2, Leipzig 1927; Bukofzer M. F., 1. c.

3 Massenkeil G., Die Wiederholungsfiguren in den Oratorien Giacomo Carissimis, AfMw 13, 1956, 42 ff.

4 Opere Complete di Carissimi a cura di Lino Bianchi, Roma, L'Istituto Italiano per la Storia della Musica. 
bereits im Jahr $1665^{5}$ ein kirchliches Verbot Carissimis Kompositionen im Druck herauszugeben. Obwohl diese Erklärung nicht ausreicht, bleibt die Tatsache bestehen, daß Carissimis Oratorien in europäischen Musikarchiven weitaus seltener erhalten blieben als andere Kompositionen des Meisters. Die Hauptquelle für Carissimis Oratorium war bis unlängst die soganannte Brossardsche Sammlung aus den Jahren 1687 - 1698 in Paris und die Chrysander-Sammlung vom Ende des 17. Jahrhunderts in der Hamburger Stadtbibliothek, die ursprünglich ebenfalls aus Frankreich kam. ${ }^{6}$ Wem diese Handschriften gedient haben, ist nicht bekannt. Abschriften der Kompositionen Carissimis aus der Zeit seines Lebens sind eine Seltenheit und seine Autographe aus San Apollinare blieben nicht erhalten, weil sie bei der Liquidierung der Jesuitenarchive im Jahr 1773 vernichtet wurden.

Deshalb gewinnt die Musikaliensammlung des Olmützer Bischois Karl Liechtenstein-Castelcorn (1664-1695) in Kroměříz (Kremsier), ${ }^{7}$ die eine Reihe zeitgenössischer Abschriften von Carissimis Oratorien enthält, erhöhte Bedeutung. Der Katalog dieser Sammlung umfaßt fast $1400 \mathrm{Kompo}$ sitionen, von denen mehr als 1000 erhalten blieben. ${ }^{8}$ Diese Kompositionen spielte man in der Kremsierer St.-Mauritz-Kollegiatkirche und auf der Bischofsresidenz. Mehr als ein Drittel hat der Leiter der bischöflichen Kapelle, der Feldtrompeter und Komponist Pavel Vejvanovský (gest. 1693), eigenhändig abgeschrieben. In der Sammlung überwiegen Komponisten, die nach dem Jahr 1600 zur Welt kamen: Pavel Josef Vejvanovský, Heinrich Schmelzer (1623-1680), Antonio Bertali (1605-1669), Philipp Jakob Rittler (1637?-1690) und Heinrich Ignaz Biber (1614-1704). Etwas schwächer vertreten sind Wolfgang Ebner (1612-1665), Giovanni Felice Sances (1600-1679) und Giacomo Carissimi (1605-1674).

Ursprünglich besaß die Liechtensteinsche Kapelle mindestens $23 \mathrm{Kom}$ positionen Carissimis, von denen 19 noch vorhanden sind. Von den nicht erhaltenen Stücken existieren nur Inventaraufzeichnungen aus dem Jahr 1695, nach denen der erzbischöfliche Archivar Anton Breitenbacher die Sammlung im Jahr 1928 ordnete. Man kann nicht ausschließen, daß es dort noch mehr Kompositionen Carissimis gab; das Inventar führt nämlich nicht bei allen Posten den Namen des Autors und außerdem ist as möglich, daß auch manche erhalten gebliebene anonyme Komposition von Carissimi stammt. In der folgenden Utbersicht nennen wir die erhaltenen und nicht erhaltenen Werke Carissimis aus Liechtensteins Sammlung in der Reihenfolge ihrer Signaturen. Bei jeder Komposition wird angeführt:

1. Ihre Ordnungszahl im vorliegenden Beitrag,

2. die Signatur,

${ }^{3}$ Ghisi F., 1. c. 797.

6 Brenet M., 1. c. $461-466$.

${ }^{7}$ Sehnal J., Die Musikkapelle des Olmützer Bischofs Karl Liechtenstein-Castelcorn in Kremsier, $\mathrm{Km}$. Jb. 51, 1967, $79 \mathrm{ff}$.

Breitenbacher A., Hudebni archiv kolegiátniho kostela sv. Mor̆ice $v$ Kroměríž (Das Musikarchiv der Kollegiatkirche St. Mauritz in Kremsier), Kroměříž 1928. 
3. der Namen der Komposition nach dem Titelblatt, bei nicht erhaltenen nach dem Inventar aus dem Jahr 1695,

4. die Besetzung nach den erhalten gebliebenen Stimmen,

5. Zeit, Ort und Kopist, sofern sie sich feststellen ließen,

6. bei bisher vielleicht unbekannten Kompositionen der Noteninzipit,

7. die Ubereinstimmung mit dem vorläufigen Verzeichnis der Werke Carissimis, das Lino Bianchi in der Beilage der oben zitierten Arbeit $F$. Ghisis (siehe Anm. 1) auf S. 803-809 veröffentlicht hat. Bei den Messen gehen wir von Massenkeils Veröffentlichung aus.9 Wir bemerken, daß die Utbereinstimmung mit Bianchi deshalb nicht genau ist, weil er bloß die Zahl der Singstimmen ohne eingehende Spezifizierung, den Namen der Komposition entweder nach ihrem Titel oder dem Textinzipit anführt, die Tonart und andere Charakteristika ganz übergeht.

1. A 249-258 Missa a quinque et a novem, trium vocem et duorum instrumentorum ... Coloniae, apud Fr. Friesem 1666. Druck. Massenkeil, 29, Nr. 12.

2. B I 28 Missa a 5 Jacomo Charissimi. Laut Inventar. Nach der Zahl der Stimmen war diese Messe offenbar mit der 11. Messe Sciolto havean dall' alte sponde identisch. Massenkeil, 29, Nr. 11.

3. B I 290 Missa a quadro di sig. Jacomo Carissimi maestro di capella in Vaticano a Rome.

Erhalten blieb bloß der Umschlag, den Vejvanovský in den achtziger Jahren des 17. Jahrhunderts geschrieben hat. Vielleicht ging es um eine Messe, die mit dem von Massenkeil in der Vatikanbücherei gefundenen Unikat identisch war. Massenkeil, 28, Nr. 1.

4. B II 130 De Tempore. Interfecto Sisara (CCCATB, Vno I II, Org.) Abschrift P. Vejvanovskýs auf Papier mährischer Provenienz etwa aus dem Jahr 1674. Notenbeispiel Nr. 1.

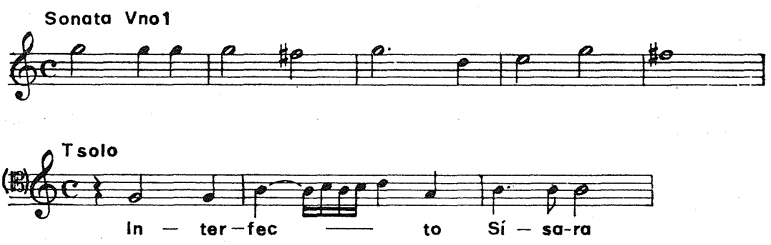

B II 131 siehe heute Nr. 19.

5. B II 132 Sicut stella. Del signor Charissimo. Aufzeichnung im Inventar.

Bianchi, Mottetti 148.

6. B II 133 Salve puellule (T, Vno I II, Gb). Abschrift eines unbekannten Kopisten auf Papier fremder Provenienz. Der Autor wird nicht genannt, aber der Name des Werks und die Besetzung stimmen mit 28 ff.

9 Massenkeil G., Ưber die Messen Giacomo Carissimis, Analecta musicol. 1, 1963, 
Bianchi, Mottetti 141 überein. Im Inventar aus dem Jahr 1695 wird diese Komposition unter anderen Werken Carissimis geführt. ${ }^{10}$

B II 134 siehe heute Nr. 18.

7. B II 160 Quo abiit dilectus meus (CA, Org). Abschrift des Kremsierer Kopisten aus der Zeit vor dem Jahr 1674. Die Vorlage kam wohl aus Rom, weil der Kopist am Ende der Orgelstimme folgende Notiz machte: A Roma dell Sig. Giacomo Carissimi, Maestro di Capella dell' Collegio Germanico et Ungarico. Notenbeispiel Nr. 2.

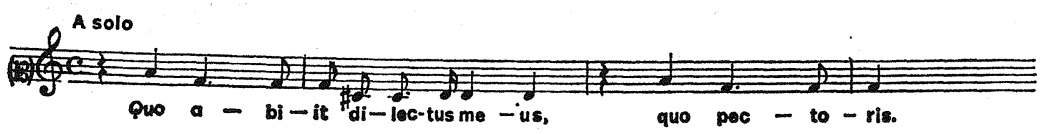

8, B II 161 De epulone. Erat vir quidam opulentissimus (CCATB 1. chori, CATB 2. chori, Vno I II, Org). Abschrift P. Vejvanovskýs und des Kremsierer Kopisten aus der Zeit um das Jahr 1680.

Bianchi, Oratori 13.

9. B II 163 Iudicium Salomonis. A solis ortu (CCATB, Vno I II, Vla ATT, Gb). Abschrift teilweise von P. Vejvanovský aus dem Jahr 1669.

Bianchi, Oratori 19.

10. B II 164 Sustinuimus pacem (CCATTB, Gb). Abschrift spätestens aus der Zeit um 1680. Das Titelblatt hat P. Vejvanovský geschrieben. Notenbeispiel Nr. 3.

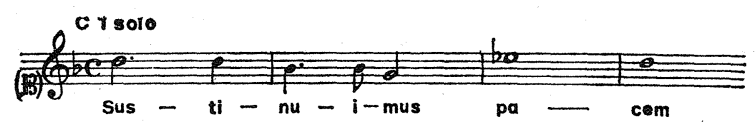

11. B II 165 Jonas: $a b 8$ vocibus. Cum repleta esset Ninive iniquitate (C I II, CATB 1. chori, CATB 2. chori, Vno I II, Org). Abschrift aus der Zeit um 1680. Das Titelblatt hat P. Vejvanovský geschrieben.

Bianchi, Oratori 17.

12. B II 166 Si linguis hominum (C I III, Vno I II, Gb, C II fehlt). Abschrift auf Papier fremder Provenienz, wahrscheinlich vor dem Jahr 1680 .

Bianchi, Mottetti 149.

13. B II 167 Proposui in mente mea quaerere et investigare. Aufzeichnung im Inventar.

Bianchi, Mottetti 124.

14. B II 168 Dialogo del Noë. Cum vidisset Deus (C I II, CCATB 1. chori, CCATB 2. chori, Vno I II, Org 1. chori). Abschrift P. Vejvanovskýs in Kremsier, November 1672.

Bianchi, Oratori, wahrscheinlich Nr. 6.

15. B II 287 Mottettum de martyribus. Usquequo peccatores (C I II III, CATB 1. chori, CAT 2. chori, Vno I II, Lto, Org; B 2. chori fehlt).

10 Vgl. auch die Bemerkung A. Breitenbachers, 1. c. 91. 
Abschrift in Kremsier, Oktober 1672. Die Orgelstimme kopierte. P. Vejvanovský. Notenbeispiel Nr. 4.

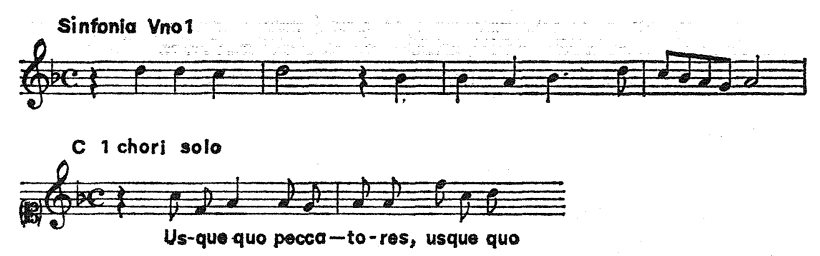

16. B II 288 Dialogo del Gigante Golia. Stabat adversus Israel (C I II, CATB 1. chori, CATB 2. chori, Vno I II, Lto, Vlne, Gb). Abschrift in Kremsier, November 1672. Die Orgelstimme schrieb P. Vejvanovsky ab.

Bianchi, Oratori vielleicht Nr. 11, Notenbeispiel Nr. 5.

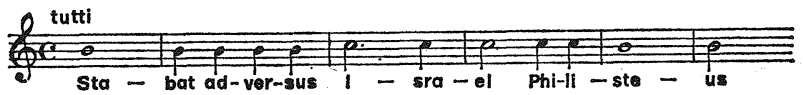

17. B II 289 Regina Hester. Persarum rex maximus Assuerus (CCATB, Vno I II, Org). Abschrift P. Vejvanovskýs um 1680. Notenbeispiel Nr. 6.

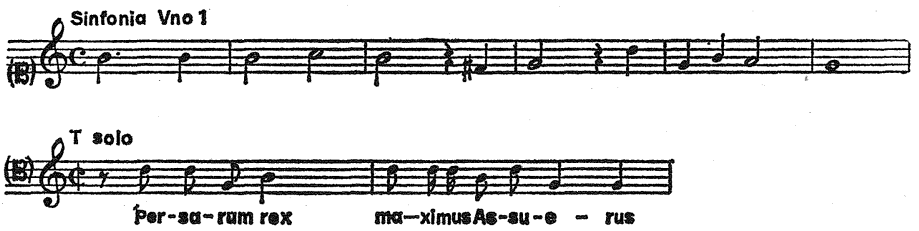

18. B II 298 De Nativitate Domini. Quasi aquila provocans ad volandum ( $T$, Vno, Vla B, Gb). Abschrift aus dem September 1674. Den ITmschlag schrieb P. Vejvanovský. Notenbeispiel Nr. 7.

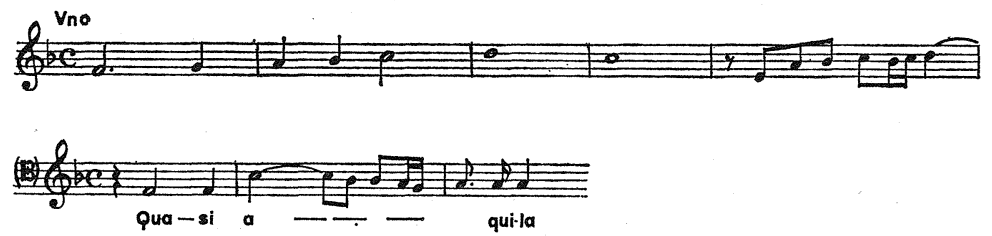

19. B II 300 Quo tam laetus (CC, Gb). Abschrift etwa aus dem Jahr 1674. Den Umschlag schrieb P. Vejvanovský.

Bianchi, Oratori 27.

20. B II 301 Turbabuntur impii (ATB, Vno I II, Lto, Org). Abschrift auf Papier fremder Provenienz.

Bianchi, Oratori 21.

21. B III 83 Dixit. (C I II III, Vno I II, Org). Abschrift des Kremsierer Kopisten aus der Zeit um 1680. 
Bianchi kennt zwei Kompositionen mit diesem Titel (Mottetti 38, 39) für 8 oder 5 Vokalstimmen(?). Notenbeispiel Nr. 8.

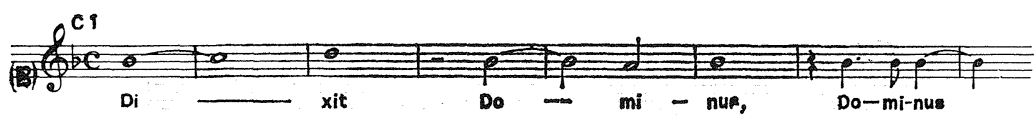

22. B III 119 Laudate pueri Dominum (Partitur enthält CCATB, Org). Abschrift P. Vejvanovskýs vom 16. 1. 1681.

Bianchi, Mottetti 83.

23. B XIV 253 Sciolto havean dall' alte sponde (Partitur enthält nur die höchste Stimme, $C$ oder $B$, und den bezifferten Baß). Abschrift eines unbekannten Kopisten auf Papier mährischer Provenienz aus den Jahren 1660-1680.

Bianchi, Composizioni su testo italiano 170. Im Vergleich mit der Edition dieses Werkes in der Gesamtausgabe läßt die Kremsierer Handschrift nur geringe Abweichungen erkennen. ${ }^{11}$

Die nachweisbar älteste Abschrift Carissimis in Kremsier ist das $J u$ dicium Salomonis, das P. Vejvanovský im Jahr 1669 für sich abgeschrieben hat. Nachdem auf dem Titelblatt als Autor ausdriicklich Don Jacomo Charissimi in Roma genannt wird, wurde diese Abschrift sicherlich unabhängig vom Druck Continuatio Theatri Musici (Herbipoli 1669) angelegt, in dem diese Komposition irrtümlich Samuel Capricornus zugeschrieben wird. Die genannte Continuatio galt bisher wegen ihres Alters für die erstrangige Quelle von Carissimis Werken. ${ }^{12}$ Vejvanovskýs Kremsierer Abschrift verweist aber den Quellenwert der Continuatio an zweite Stelle.

Die Provenienz der Abschriften von Carissimis Kompositionen wurde noch nicht geklärt. Nur die unter Nr. 6, 12, 20 angeführten Abschriften sind sicher nicht in Mähren entstanden. Sie wurden nämlich von einem unbekannten Kopisten auf Papier der Marke Fabriano geschrieben, das man im ganzen 17. Jahrhundert in Mittel- und Süditalien verwendete.13 In Liechtensteins Sammlung gibt es keine andere auf diesem Papier geschriebene Komposition. Deshalb darf man annehmen, daß die erwähnten drei Kompositionen in Italien abgeschrieben und nach Mähren gesandt wurden.

Unmittelbare Musikkontakte mit Rom lassen sich am Beginn von Liechtensteins Episkopat nachweisen. Im Laufe seines Romaufenthalts komponierte nämlich der Dekan von Strážnice Mikuláš František Faber ein Threnon musicum genanntes opus zum Andenken an den Tatareneinfall in Mähren 1663, den er offenbar selbst miterlebt hatte. Als er erfuhr, Liechtenstein sei zum Olmützer Bischof gewählt worden, widmete er ihm am 16. Juni 1664 in Rom diese Komposition und ließ sie im selben Jahr

${ }^{11}$ Carissimi G., Cantate Vol. 1, a cura di L. Bianchi, Roma 1960.

12 Rybarič R., Judicium Salomonis, Samuel Capricornus a Giacomo Carassimi, Musicol. Slovaca 3, 1972, 170. 7628.

13 Briquet C. M., Les filigranes, ed. by A. Stevenson, Amsterdam 1968, No. 
in Wien bei M. Cosmerovius drucken. Es ist nicht bekannt, weshalb und wie lange sich Faber in Rom aufhielt; wahrscheinlich wohnte er im Collegium Germanicum und hat dort bestimmt das Schaffen des berümten Carissimi kennengelernt. Einflüsse dieses Komponisten treten übrigens auch bei seinem Threnon musicum zutage. Mit der Widmung dieser Komposition verfolgte er offenbar das Ziel einen besseren Posten zu gewinnen. Sein Wunsch ging in Erfüllung, denn am 5. Dezember wurde Faber Dekan des Kollegiatkapitels und Pfarrer zu St. Maria in Kremsier. Diese Funktionen bekleidete er bis zu seinem Tod im Jahr 1673. Aus seiner Kremsierer Zeit blieben zwar merkwürdigerweise keine Belege einer Musikaktivität erhalten, angesichts seiner musikalischen Interessen kann man jedoch die Möglichkeit nicht ausschließen, daß er Carissimis Kompositionen vermittelte. ${ }^{14}$

Geschäftsverbindungen mit Italien unterhielt der Bischof über seinen römischen Agenten Giovanni Petignieri. Aus der Korrespondenz ist bekannt, daß Petignieri dem Bischof verschiedene erlesene Waren, nicht aber Musikalien verschaffte, abgesehen vom Jahr 1666, als er in Venedig notierte liturgische Bücher kaufte. Es ist deshalb wenig wahrscheinlich, daß gerade er Carissimis Kompositionen für die bischöfliche Kapelle beschafft hätte.

Nachdem der Großteil von Carissimis Kompositionen für Kremsier in Mähren abgeschrieben wurde, mußte im Bereich der Bischofsresidenz noch ein anderes Musikzentrum existieren, das diese Kompositionen besaß. Wir sahen, daß sich der bischöfliche Kapellmeister Pavel Vejvanovský an einer Reihe von Kremsierer Kopien beteiligte, sie wurden also entweder an Orten abgeschrieben, die Vejvanovský besuchte oder man lieh ihre Vorlagen nach Kremsier zur Abschrift. Pavel Vejvanovský hielt sich am häufigsten in Kremsier und auf den Gütern des Bischofs in Vyškov, Mírov, Svitavy auf, besuchte dienstlich auch Brünn und besonders Olmütz. Und gerade in Olmütz, dem Zentrum des Bistums, lag ein bedeutendes Jesuitenkollegium mit einer Universität und einem Musikseminar. In der ersten Hälfte seines Episkopats (etwa bis ins Jahr 1680) war Bischof Karl Liechtenstein den Jesuiten sehr gewogen, verwendete gern ihre Dienste bei gegenreformatorischen Aktionen und versuchte sie sogar nach Kremsier zu führen. Nach dem Jahr 1680 kühlte sich die Beziehung ab und der Bischof orientierte sich auf die Piaristen. Carissimis Kompositionen wurden jedoch zum Großteil in den siebziger Jahren abgeschrieben, als die Beziehungen des Bischofs zu den Jesuiten noch herzlich waren.

Uber das Musikleben bei den Olmützer Jesuiten im 17. Jahrhundert ist bisher nur wenig bekannt. Erst aus dem 18. Jahrhundert sind wir genauer. über die Musiker des Jesuitenseminars und die großartigen, öffentlich zugänglichen Oratorienaufführungen informiert, die sie in Olmütz veranstalteten. Weder aus dem 17. noch aus dem 18. Jahrhundert blieb aber ein

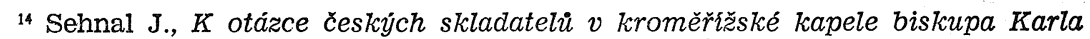
Liechtensteina-Castelcorna (Zur Frage der tschechischen Komponisten in der Kremsierer Musikkapelle des K. Liechtenstein-Castelcorn), Umění a svět 1, 1957, $39 \mathrm{ff}$. 
Musikalienverzeichnis der Olmützer Jesuiten erhalten, das Informationen über das Musikrepertoire bringen könnte. Der Mangel an Quellen kann an sich keine Zweifel daran erwecken, daß bereits im 17, Jahrhundert die Musikpflege am Olmützer Jesuitenkollegium auf einem hohen Niveau stand. Die Jesuiten mochten am ehesten Kompositionen aus dem Collegium Germanicum erhalten haben, trotz dem päpstlichen Verbot, das übrigens vielleicht nicht einmal streng eingehalten wurde. Sicher zögerten die Olmützer Jesuiten nicht, dem bischöflichen Kapellmeister Kompositionen ihres römischen Maestro zur Abschrift zu leihen, wenn ihnen an guten Kontakten mit dem Bischof gelegen war. Und Pavel Vejvanovský besaß zu den Jesuiten ebenfalls von Jugend an enge Beziehungen: hatte er doch in den Jahren 1656-1660 an ihrem Gymnasium in Opava (Troppau) ${ }^{15}$ studiert und ließ auch seinen Sohn Jan Karel ${ }^{15}$ bei den Olmützer Jesuiten studieren.

Die Kaiser Ferdinand III. und Leopold I. versuchten Carissimi für ihre Wiener Hofkapelle zu gewinnen, mit deren Mitgliedern auch der Olmützer Bischof korrespondierte. Trotzdem enthält nicht einmal diese Korrespondenz eine Andeutung, daß er sich für Carissimi interessiert hätte. Man kann auch nicht beweisen, daß die in Kremsier erhalten gebliebenen Kompositionen Carissimis zu dieser Zeit auch in Wien bekannt waren. Pavel Vejvanovský besuchte mehrmals Wien (beispielsweise in den Jahren 1661 und 1665), und schrieb dort jedesmal eine Menge Kompositionen ab, aber keine einzige von Carissimi. Wien war also kaum jener Ort, wo man die Vorlagen für die Kremsierer Kopien der Kompositionen dieses Meisters erhielt. Das Olmuitzer, vielleicht auch das Brünner Jesuitenkollegium scheinen desshalb die einleuchtendsten Verbindungsglieder zwischen dem Collegium Germanicum und der Kremsierer Bischofskapelle gewesen zu sein.

Von den Messen, Psalmen und Serenaden I naviganti abgesehen, sind alle Kompositionen Carissimis in Kremsier im zweiten, Offertoria bezeichneten Abschnitt des Verzeichnisses zu finden. Dieser Abschnitt umfaßt geistliche, größtenteils zu nichtliturgischen lateinischen, und nur ausnahmsweise deutschen Texten komponierte Werke, die sich auf die einzelnen Abschnitte und Feiertage des Kirchenjahres beziehen. Viele tragen im Titel die Bezeichnung Mottettum oder Offertorium, man kann also annehmen, daß sie meist während des Messe-Offertoriums aufgeführt wurden. Dieselbe Aufgabe erfüllten in Kremsier jene Kompositionen Carissimis, die allgemein als Oratorien gelten (Nr. 4, 8, 9, 11, 14, 16, 17). Vorläufig fehlt jeder Grund zur Annahme, daß diese oder ähnliche Kompositionen in Kremsier selbständig, und nicht an die Liturgie gebunden, erklungen wären. Solche Kompositionen hätten sicherlich im Inventar aus dem Jahr 1695 eine eigene Gruppe gebildet, ähnlich wie beispielsweise die Vertonungen des Salve Regina, Te Deum u. a.

\footnotetext{
15 Breitenbacher A., 1. c. 80.

${ }^{16}$ Sehnal J., Jména hudebnikú 17. století $v$ matrice olomoucké university (Musiker des 17. Jh. in der Matrikel der Olmützer Universität), Zprávy Vlastiv. ústavu v Olomouci 127, 1966, 10.
} 
Außer Nr. 22 und 23 sind alle Kompositionen Carissimis in Kremsier in sorgfältig abgeschriebenen Stimmen zu je einem Exemplar erhalten. Der Generalbaß ist mit Organo überschrieben und trägt keine Bezeichnung. Er ist immer eingehend beziffert, wie es für Carissimi typisch war. ${ }^{17}$ Es ist nicht sicher, ob die Baßmelodie immer durch das Baßstreichinstrument verdoppelt wurde, weil der selbständige Violone-Part in Nr. 16 nur für die tutti des Schlusses ausgeschrieben ist. Die Baßviola besitzt in der Sonate Nr. 18 den Charakter der zweiten melodischen Stimme, verdoppelt aber in den Vokalpartien die Melodie des Generalbasses. In Nr. 15, 16, 20 existiert neben der Orgelstimme noch die Leuto überschriebene Stimme für Baßlaute, die ebenso notiert ist wie der Orgelpart. Von der Orgelstimme unterscheidet sie sich durch die zeitweilige Teilung der längeren in kürzere Werte, und dadurch, daß sie blo $\beta$ in den Sinfonien und dem tutti des Schlusses auftritt. Das überrascht deshalb, weil die Laute an solchen Stellen kaum zu Wort kommen konnte. Die einzige Erklärung wäre, daß die Stimme von mehreren Lautenspielern gespielt wurde. Darauf sollte auch der Umstand hinweisen, daß das Leuto in Nr. 16 mit zwel Exemplaren vertreten ist. Neben einer einzigen Messe Bertalis und der anonymen Komposition Hercules et Omphale sind Carissimis Werke die einzigen der Liechtensteinschen Sammlung, die einen Lautenpart enthalten. Selbständige Stücke für dieses Instrument blieben in Kremsier nicht erhalten, obwohl der Bischof bekanntlich im Jahr 1676 in Wien eine Laute bestellt hat. ${ }^{18}$ Als Generalbaßinstrument hat man sie jedoch in Kremsier offenbar auch bei der Kirchenmusik verwendet. Im großen und ganzen ist die Instrumentalkomponente von Carissimis Kompositionen in Kremsier dieselbe wie in seinen anderen Werken: 2 Violinen und continuo. Nur ausnahmsweise gesellen sich zu diesem Ensemble die Laute und in einem Fall drei Violen (Nr. 9), die nur in den Instrumentalabschnitten und im Schlußtutti spielen.

In unmittelbarer Nähe von Kremsier, in Tovačov, unterhielt Graf Salm eine Kapelle, die ein ähnliches Repertoire wie die Kremsierer Kapelle aber nur nach dem Inventar aus dem Jahr 1699 bekannt sind.19 Sie waren dort unter der Rubrik Concertus eingereiht:

Exultate $\grave{a} 3$ ex F, 2 Canti solo (bei Bianchi nicht angeführt),

Quo tam laetus à 3 ex G(!), 2 Canti solo (Bianchi, Oratori 27, siehe auch Kremsier Nr. 19),

Confitebimur $\grave{a} 5$ ex $C \grave{a} 4$ Cantis (bei Bianchi nicht angeführt),

Virgo Davidica à 4 ex $C$, Canto solo (bei Bianchi nicht angeführt),

Sacris dicata à 3 ex G, 2 Canti solo (bei Bianchi nicht angeführt),

Cantemus Domino à 3 ex G, 2 Canti (bei Bianchi nicht angeführt).

17 Darauf hat schon M. Brenet, 1. c. 482 hingewiesen.

"Nettl P., Die Wiener Tanzkomposition in der 2. Hälfte des 17. Jh., Studien z. Musikwiss. 8, 1921, 173.

19 Racek J.; Inventár hudebnín tovačovského zámku z konce 17. století (Inventar der Musikalien des Schlosses Tovačev aus dem Ende des 17. Jh.), Musikologie 1, 1938, 62 fí. 
Die Tatsache, daß nur eine dieser sechs Kompositionen Bianchi bekannt war und auch nur eine einzige in Kremsier belegt ist, deutet abermals darauf hin, daß es in Mähren noch irgendeine ausgiebige Quelle von Carissimis Kompositionen gegeben haben muß. Daß diese Quelle in Olmütz zu suchen ist, bestätigt schon der Umstand, daß Tovačov etwa auf dem halbem Weg zwischen Kremsier und Olmütz liegt.

Während Carissimis Handschriften aus Tovačov nicht erhalten blieben, befindet sich eine seiner Kompositionen in der Sammlung der städtischen St.-Jakob-Pfarrkirche zu Brünn: ${ }^{20}$ Cantus de Ss. Eucharistia (Cum audisset Gedeon), für C und Gb. Die Vokalstimme wurde schon am 12. 8. 1678 abgeschrieben und die Komposition gehörte ursprünglich dem Math. Fr. Altmann, einem großen Verehrer J. J. Fuxens, der in den Jahren 1682-1715 Kantor an der Brünner St.-Peter-und-Paul-Kirche war. Notenbeispiel Nr. 9.

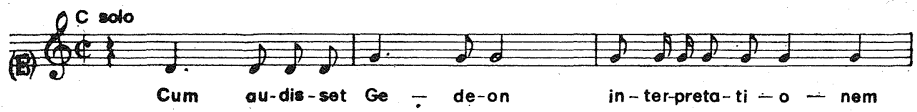

Interessanterweise kennen die übrigen bisher bekannten mährischen Musikinventare aus dem 17. Jahrhundert (Kroměříž 1659, Litovel 1672, Strážnice 1675) Carissimis Namen nicht.

Auch in Böhmen waren seine Kompositionen bekannt, allerdings weniger verbreitet als in Mähren. Unseres Wissens blieb dort keine einzige erhalten, man kennt sie nur aus Inventaren. Musikinventaren des Piaristenkollegiums in Slaný aus dem Jahr 1713:21

Omnes sancti quanta passi sunt à $2 \mathrm{C}$ et Org. Gewonnen vor $1668 \mathrm{Bian-}$ chi, Mottetti 104.

Domine Deus meus à $2 C$ et Org. Gewonnen 1692. Bianchi, Mottetti 42 bringt à 1 (!).

Die Piaristen in Slaný standen bekanntlich mit Kremsier und Olmütz in Verbindung, denn in ihrem Inventar erscheinen aus Kremsier bekannte Komponisten und Kompositionen. Inventar der ehemaligen Eggenbergschen Kapelle in Böhm. Krumau aus dem Jahr 1706:22

Gaudete Pastores CATB, 4 Instr., Org. Bianchi, Mottetti 65 kennt eine gleichnamige Komposition à 3 (!),

Iudicium Salomonis ATB (!), 6 Instr., Org. Bianchi, Oratori 20, auch Kremsier Nr. 9.

Musikinventar des Piaristenkollegiums in Kosmonosy aus dem Jahr $1712: 23$

${ }^{20}$ Die Komposition befindet sich im Institut für Musikgeschichte des Mährischen Museums in Brno, Sign. A 1555.

${ }^{21}$ Inventarium Rerum et Instrumentorum Musicalium Chori Slanensis Schol. Piar... 1713. Musikabt. des Nationalmuseums in Prag.

${ }^{22}$ Komplex der Musikinventare der ehemaligen Eggenbergschen Musikkapello in Böhm. Krumau, Staatsarchiv Třebon̆, Dienststelle Böhm. Krumau, Sign. $1283 / 10-64$.

${ }_{23}$ Culka Z., Inventár̆ hudebních nástrojů a hudebnin piaristické koleje $v$ Kosmonosich (Inventar der Musikinstrumente und Musikalien des Piaristenkollegiums in Kosmonosy), Příspěvky $\mathrm{k}$ dějinám české hudby 2, 1972, 22. 
O felix anima. CA. Bianchi Mottetti 99.

Musikinventar des Zisterzienserklosters in Osek aus dem Jahr 1720:24

Audite mortales de Nativitate Domini. Bianchi, Mottetti 2 kennt Adeste (!) mortales.

Die geringere Zahl der Belege von Carissimis Schaffen in Böhmen läßt sich teilweise mit dem Umstand erklären, daß die genannten böhmischen Inventare jünger sind als die mährischen und im Durchschnitt Musikalien enthalten, die 30-40 Jahre vor ihrer Niederschrift gewonnen wurden und gewiß Wert auf Kompositionen legen, die zur Zeit der Inventarisierung lebendig und aktuell waren. Alle angeführten Belege gestatten den Schluß, daß die Beliebtheit von Carissimis Schaffen in unseren Ländern zwischen den 60 . und 80. Jahren des 17. Jahrhunderts gipfelte. Gegen Ende des 17. Jahrhunderts hatte die Musik dieses Komponisten bereits ihre Zugkraft verloren und wurde nach 1700 wahrscheinlich kaum mehr gespielt; sie war veraltet. Wenn Carissimi noch nach diesem Zeitpunkt bei uns beliebt gewesen wäre, hätte sich das gewiß zumindest in der Musikaliensammlung bei St. Jakob in Brün und in den Inventaren von Kosmonosy und Osek geäußert. Sicher wäre sein Name auch bel den Benediktinern in Rajhrad aus dem Jahr $1725^{25}$ erschienen, geschweige denn vom Inventar des Jesuitenkollegiums in Uherské Hradište aus dem Jahr $1730 .^{26}$ Wir wollen nicht ausschließen, daß Carissimi bei uns schon vor dem Jahr 1660 bekannt war, können dies aber nicht beweisen. Das reiche Vorkommen von Schlußhemiolen in den Liedern des Adam Michna z Otradovic (1600-1676) könnte der Abglanz einer beliebten Manier des römischen Meisters gewesen sein, aber auch irgendeines anderen italienischen Komponisten dieser Zeit. ${ }^{27}$ Es gibt eine bisher unbelegte HypoMichna z Otradovic), Hudebni věda 12, 1975, 19.

these, Adam Michna habe im Jahr 1638 Rom mit einer Botschaft des Fürsten Johann Anton Eggenberg besucht und Gelegenheit gehabt, Carissimis Werke kennenzulernen. Viel stärker war wohl der Einfluß von Michnas Kontakten mit den Jesuiten aus Jindřichův Hradec, über deren Musik leider nichts Näheres bekannt ist. Das rhetorische Pathos der biblischen Gestalten Carissimis ist sicher nicht ohne Einfluß auf das geistliche Schaffen der Komponisten geblieben, die mit Carissimis Werk in Berührung kamen (Vejvanovský, Rittler u.a.), darüber wird man aber erst nach Analysen ihrer Kompositionen mehr sagen können.

Lange galt Carissimi in den Böhmischen Ländern als Autorität der Musiktheorie. Noch an der Wende vom 17. zum 18. Jahrhundert mußte bei uns sein Handbuch Ars cantandi in der deutschen Ubersetzung Leichte Grund-Regeln zur Sing-Kunst (Augsburg 1689) zu haben sein. Einen Beleg

${ }^{24}$ Catalogus musicaliorum anno 1720 in Ordinem digestus... Musikabt. des Nationalmuseums in Prag.

${ }^{25}$ Straková Th., Rajhradský hudební inventár z roku 1725 (Das Musikinventar von Rajhrad aus dem J. 1725), Čas. Mor. musea-vědy spol. 58, 1973, 217 ff.

${ }^{26}$ Sehnal J., Hudba $v$ jesuitském seminár $i$ v Uherském Hradišti $v$ roce 1730 (Die Musik im Jesuitenseminar von Uherské Hradiště im J. 1730), Hudební věda 4, 1967, 139 ff.

27 Sehnal J., Písně Adama Michny z Otradovic (1600-1676) (Lieder des Adam Michna z Otradovic), Hudební věda 12, 1975, 19. 
dafür bringt Tomáš Baltazar Janovka (geb. 1669 in Kutná Hora, gest. 1741 in Prag),28 in seinem Musiklexikon Clavis ad thesaurum magnae artis musicae (Prag 1701), das Carissimi nach A. Kirchner29 am häufigsten zitiert und manche Abschnitte aus Carissimis Schriften wörtlich übernimmt. Weil in tschechischen Bibliotheken kein einziges Exemplar von Carissimis

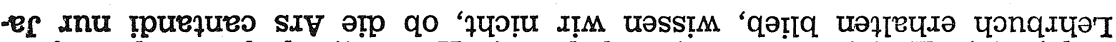
novka oder auch breiteren Kreisen bekannt gewesen ist. Es ist sicher bemerkenswert, da $\beta$ der tschechische Musiktheoretiker zur Propagierung von Carissimis Namen in einer Zeit beigetragen hat, als der Stil seiner Musik bereits ïberholt war.

Die Pflege der weltlichen Kompositionen Carissimis entsprach offenbar der bei uns damals relativ geringen Frequenz der weltlichen Vokalmusik überhaupt. Anonym blieb in Kremsier die Serenada I Naviganti (Nr. 23) erhalten und wirkt als Kuriosum, weil es die einzige Komposition dieser Art ist. Eine Art verspätete Reprise Carissimis war vielleicht das "scherzhafte Terzett Barba Capucinorum", das die Fundatisten des Augustinerklosters zu Altbrün im Rahmen des collegium musicum am 9. 12. 1817 aufführten. ${ }^{29}$ Der Autor dieses musikalischen Scherzes ist zwar nicht genannt, aber Name und Besetzung des Stücks erinnern auffallend an Carissimis Venerabilis barba Capucinorum (Bianchi, Plaisanteries 3). Nachdem es um eine komische Szene ging, störte es offenbar nicht, daß die Kompositionsmittel längst überholt waren; gerade das Anachronistische mußte sie der spätjosefinischen Gesellschaft in einer Zeit der keimenden Romantik besonders witzig erscheinen lassen.

Ich bemühte mich zu zeigen, daß Carissimis Schaffen in der zweiten Hälfte des 17. Jahrhunderts in den Böhmischen Ländern bekannt war. In der Nähe von Kremsier und seiner Kapelle mußte in Mähren noch ein anderes Musikzentrum existieren, das mit Carissimis Kompositionen besser versorgt war als manches andere europäisches Musikzentrum. Die Existenz eines solchen Zentrums beweisen vor allem die zahlreichen wertvollen Abschriften von Carissimis Kompositionen in der Sammlung der Kapelle des Olmützer Bischofs aus den Jahren 1600-1690. Anscheinend hat dann Carissimis Musik im letzten Jahrzehnt des 17. Jahrhunderts in Böhmen und Mähren ihre Anziehungskraft verloren und der Name des Komponisten überlebte nur mehr im Musiklexikon des Tomáš Baltazar Janovka.

\section{POVZETEK}

Delo Giacoma Carissima (1605-1674) je bilo v drugi polovici 17. stoletja dobro znano $v$ ceških deželah, o tem pričajo skladbe tega ustvarjalca, ki so $\mathrm{v}$ ceških glasbenih zbirkah oz. glasbenih inventarjih. V tej zvezi so zlasti važni rokopisi v glasbeni zbirki olomouškega škofa Karla Liechtensteina-Castelcorna v Kroměříŭu.

${ }^{28}$ Die Lebensdaten Janovkas hat T. Volek entdeckt. Vgl. seine Arbeit Tomás Baltazar Janovka, Představitel české barokni a hudebni vadelanecké tradice (T. B. Janovka - Repräsentant der Tradition des tschechischen musikalischen Barock aus dem gebildeten Bürgertum), Hudební věda 9, 1972, 344 ff.

${ }_{29}$ Sehnal J., Die Bläserharmonie des Augustinerklosters in Altbrünn, Sborník prací fil. fak. brnĕnské university H. 8, 1973, 131. 
Tu so mnogi unikati skladb navedenega komponista, nekateri sodijo med najsta. rejše ohranjene prepise nasploh. Večino teh prepisov je oskrbel Pavel Vejvanovský, deloma tudi tamošnji kopisti. Avtor prispevka domneva, da je razen Kroměřiža obstajal na Moravskem še nek glasbeni center, ki je imel neposredne stike z Ri. mom in $\mathrm{s}$ tem $\mathrm{z}$ ustanovo, $\mathrm{v}$ kateri je deloval Carissimi (Collegium Germanicum). Ta center je najbrž bil jezuitski kolegij $\mathrm{z}$ glasbenim seminarjem $\mathrm{v}$ Olomoucu. Kaže, da so Carissimijeve skladbe v Kroměřížu iz dobe, ko je škof vzdrževal z olomouškimi jezuiti tesne zveze. Prvi propagator del tega skladatelja je utegnil biti v Kroměřížu skladatelj Mikuláš František Faber, ki je bil v letu 1664 v Rimu, v letih 1665-1673 pa dekan kolegijskega kapitlja v Kroměřížu. Konec 17. stoletja preneha aktualnost Carissimijeve glasbe $\mathrm{v}$ čeških deželah. Kljub temu je veljal Carissimi še naprej za avtoriteto $\mathrm{v}$ teoriji glasbe, gotovo po zaslugi $\mathrm{T}$. B. Janovke Clavis ad thesaurum magnae artis musicae (Praga 1701), ki je gradivo izdatno črpal iz priročnika Ars cantandi G. Carissima. 